\title{
Investigación sobre Tasas de Morbi-mortalidad Perinatal en Barrios Periféricos de la Ciudad de Ibagué
}

\author{
Dres. Ramiro Lozano Neira*, Héctor Mancera**, \\ Marina de Camacho***, Josefina de Carvajal**** $y$ \\ Jorge $\operatorname{Vargas} * * * *$
}

\section{SOCIEDAD TOLIMENSE DE OBSTETRICIA Y GINECOLOGIA SECRETARIA DE SALUD DEL TOLIMA}

\section{INTRODUCCION Y OBJETIVOS}

El estudio, cuyos hallazgos se presentan, constituye un importante avance en el conocimiento de lo que actualmente es la situación de la madre en la ciudad de Ibagué.

Por primera vez se ha recolectado información intra-domiciliaria, casa a casa, en una muestra representativa de la población de estrato social bajo y medio.

* Jefe Servicio Salud del Tolima

* Jefe Sección Materno Infantil

*** Enfermera Jefe Sección Materno Infantil.

**** Directora Escuela Auxiliar de Enfermería Jefe Estadística
Estos datos representan los intereses aunados de la Sociedad Tolimense de Obstetricia y Ginecología y la Secretaría de Salud del Tolima.

\subsection{Objetivo general}

Hacer un diagnóstico de la situación actual con relación a la atención materno infantil en Ibagué, a través de la recolección y análisis de la información de las pacientes en una muestra vivienda a vivienda, teniendo en cuenta variables socio-demográficas y atención médica específica, a través de una serie de datos consignados en un formulario especial y con un criterio unificado por la Sociedad Vallecaucana de Obstetricia y Ginecología. 


\subsection{Objetivos Específicos}

1.2.1 Establecer parámetros reales de la salud materno infantil por zona.

1.2.2 Propender por que los beneficios de los programas materno infantiles actuales y futuros se evalúen midiendo su impacto sobre la morbi-mortalidad de la comunidad.

\section{DESCRIPCION SISTEMA SALUD DEL TOLIMA}

El Departamento del Tolima tiene cinco Unidades Regionales, cuyos Hospitales sedes están ubicados en los Municipios de Ibagué, Armero, Espinal, Líbano y Chaparral.

\subsection{Unidad Regional de Ibagué: Area de influencia}

Esta Regional cuenta con siete Hos pitales locales y dos Centros de Salud, su sede es el Hospital Regional "Federico Lleras Acosta" en la ciudad de Ibagué. (Anexo: No. 1).

Municipios

Ibagué:

Alvarado (C.S.):

Anzoátegui:

Cajamarca:

Piedras (C.S.):

Roncesvalles:

Rovira:

Santa Isabel:

Valle de San Juan:

Venadillo:

IBAGUE: En la zona urbana de ibagué hay 15 Centros de Salud, con servicio de consulta externa general y actividades de Enfermería. (Ver Anexo: No. 2).
De estos organismos de salud, se seleccionaron cuatro Centros de Salud para hacer el estudio y cuentan con el siguiente personal:

UPA. Uribe Uribe: 1 Médico con 3 horas.

1 Auxiliar de Enfermería.

2 Promotoras de Salud.

UPA. El Bosque: 1 Médico con 3 horas.

1 Auxiliar de Enfermería.

1 Promotora de Salud.

UPA. La Gaviota: 1 Médico con 4 horas.

1 Auxiliar de Enfermería.

3 Promotoras de Salud.

C.S. Jordán:

3 Médicos con 2 horas cada uno.

2. Auxiliares de Enfermería.

2 Odontólogos.

C.S. Jordán II:

1 Médico con 4 horas.

1 Auxiliar de Enfermería.

1 Odontólogo.

\section{MATERIAL Y METODOS}

3.1 Para efectos del trabajo realizado sobre tasas de morbi-mortalidad perinatal se tomó la población de Ibagué, que es de 244.681 habitantes de acuerdo a datos estimados para 1981.

El porcentaje de habitantes para la zona urbana es de $86.03 \%$ que equivale a 210.585 habitantes. 
Si. tomamos un promedio de 6 habitantes por vivienda, nos da un número total de viviendas de 35.098 .

De acuerdo a lo convenido en la reunión realizada en Ibagué con la Coordinadora de la investigación, se tomó un $8 \%$ del número total de viviendas que corresponde a 1.867 viviendas.

3.2 El sector que se escogió para realizar el trabajo sobre tasas de morbimortalidad perinatal, se distribuyó de la siguiente forma:

3.2.1 Para la selección del sector en el cual se aplicaron las encuestas casa a casa se tuvieron en cuenta las siguientes características:

3.2.1.1 Se hizo el análisis socioeconómico y cultural, siendo el deseo de llevar dos sectores con características socio-económicas y culturales diferentes.

3.2.1.2 Se tuvo en cuenta en la selección del área el recurso humano disponible (Auxiliares, Ayudantes de Enfermería, etc.).

3.2.2 Los sectores escogidos de acuer-do a los parámetros anteriores son los siguientes, con sus respectivas características:

\subsubsection{Clase Baja.}

3.2.2.1.1 Barrio Uribe Uribe con 300 viviendas.

3.2.2.1.2 Barrio La Gaviota con 728 viviendas.

3.2.2.1.3 Barrio El Bosque con 459 viviendas. Para un total de 1.487 viviendas.

\subsubsection{Clase Media}

3.2.2.2.1 Barrio El Jordán con 1.321 viviendas.

3.2.2.3 Para un gran total de viviendas de 2.867 .

3.2.3 Para la realización de la investigación sobre tasas de morbi-mortalidad perinatal se cuenta con el siguiente recurso humano:

3.2.3.1 Sociedad de Ginecología y Obstetricia del Tolima, representada por dos Coordinadores.

3.2.3.2 Servicio Seccional de Salud representado así: Jefe de Servicio de Salud del Tolima, Director General Hospital Regional "Federico Lleras Acosta", Jefe Sección Materno Infantil, Enfermera Jefe de la Sección Infantil, Jefe Sección Información Servicio de Salud, Enfermera Jefe de le Regional de Ibagué, Enfermera Jefe de Unidades Primarias de Salud.

\subsubsection{Nivel Operativo}

Promotores de Saneamiento Ambiental, Auxiliares de Enfermería, Ayudantes de Enfermería y Promotoras.

\subsection{Características sanitarias de la población.}

3.4.1 Clase Baja: (Ver Cuadro No. 1).

Cuadro que nos muestra las características desventajosas de la población de clase baja analizada, donde el 33\% del total de viviendas están sin alcantarillado, $75 \%$ habitan viviendas no higiénicas, $33 \%$ no tienen inodoro y $9 \%$ tienen letrinas. Las vías de penetración están en pésimas condiciones, son sitios de botaderos de basuras y no hay recolección de basuras por parte de las Empresas Municipales. Existen zonas tuguriales 
Cuadro No. 1

\begin{tabular}{|l|c|c|c|c|c|c|c|}
\hline \multirow{2}{*}{ Barrios } & \multicolumn{2}{|c|}{ alcantarillado } & \multirow{2}{*}{ Acueducto } & \multicolumn{2}{c|}{$\begin{array}{c}\text { Vivienda hi- } \\
\text { giénica }\end{array}$} & \multicolumn{2}{c|}{ inodoro } \\
\cline { 2 - 5 } & CON & SIN & & SI & NO & SI & NO \\
\hline Bosque & $32 \%$ & $68 \%$ & $90 \%$ & $10 \%$ & $90 \%$ & $33 \%$ & $59 \%$ \\
\hline $\begin{array}{l}\text { Uribe } \\
\text { Uribe }\end{array}$ & $85 \%$ & $15 \%$ & $100 \%$ & $28 \%$ & $72 \%$ & $70 \%$ & $20 \%$ \\
\hline La Gaviota & $85 \%$ & $15 \%$ & $100 \%$ & $35 \%$ & $65 \%$ & $70 \%$ & $20 \%$ \\
\hline
\end{tabular}

como la Playa, en el Barrio el Bosque con 2.000 habitantes $y$ el Barrio Uribe Uribe con 1.200 habitantes.

\section{ANALISIS}

\subsection{Distribución número de familias por} casa. Total de personas por casa (Promedio).

Se informan los resultados del estudio en 2.867 familias que corresponde al $8 \%$ de la población total de 15.412 habitantes, con un promedio de 5.4 personas por familias (Tabla No. 1).

\subsection{Distribución por grupos de edad y sexo}

El mayor número de personas está entre los 15 a 44 años de edad $(45 \%)$, siendo predominante el número de mujeres en edad reproductiva, $3.788(24.6 \%)$, sobre el de hombres $3.209(20.8 \%)$, seguido por el grupo de 1 a 4 años, $6.050(39.3 \%)$.

Tabla No. 1

\begin{tabular}{|c|c|c|c|c|c|c|}
\hline \multirow{2}{*}{$\begin{array}{c}\text { Grupos de } \\
\text { Edad }\end{array}$} & \multicolumn{2}{|c|}{ Hombres } & \multicolumn{2}{c|}{ Mujeres } & \multicolumn{2}{c|}{ Total } \\
\cline { 2 - 7 } & NO & $\%$ & NO & \% & NO & $\%$ \\
\hline$<1$ & 233 & 1.5 & 234 & 1.5 & 467 & 3.0 \\
$1-14$ & 2.973 & 19.3 & 3.077 & 20.0 & 6.050 & 39.5 \\
$15-44$ & 3.209 & 20.8 & 3.788 & 24.6 & 6.997 & 45.4 \\
$45 y+$ & 920 & 6.0 & .978 & 6.3 & 1.898 & 12.3 \\
\hline Total & 7.335 & 47.6 & 8.077 & 52.4 & 15.412 & 100.0 \\
\hline
\end{tabular}


4.3. Mortalidad por grupos de edad y sexo

Tabla No. 2

\begin{tabular}{|l|c|c|c|}
\hline \multirow{2}{*}{$\begin{array}{l}\text { Grupos de } \\
\text { edad }\end{array}$} & \multicolumn{2}{|c|}{ Sexo } & \multirow{2}{*}{ Total } \\
\cline { 2 - 3 } & Hombres & Mujeres & \\
\hline$<^{1}$ & 51.5 & 21.4 & 36.4 \\
$1-14$ & 1.0 & 1.0 & 1.0 \\
$15-44$ & 3.1 & 0.8 & 1.9 \\
$45 y+$ & 19.6 & 8.2 & 13.7 \\
\hline Total & 5.9 & 2.4 & 4.0 \\
\hline
\end{tabular}

La mortalidad más alta está entre los menores de un año, $36.4 \times 1.000$, sobresaliendo el grupo de hombres, $51.5 \times$ 1.000 , que muestra una relación de 2.4 a 1 (Tabla No. 2).

\subsection{Mortalidad ( $10^{3}$ Hab.). Por grupos de edad y sexo}

En la población estudiada se encontraron 17 muertos en menores de un año,

Tabla No. 3

\begin{tabular}{|l|c|c|c|}
\hline \multirow{2}{*}{$\begin{array}{l}\text { Grupos de } \\
\text { edad }\end{array}$} & \multicolumn{2}{|c|}{ Sexo } & \multirow{2}{*}{ Total } \\
\cline { 2 - 4 } & Hombres & Mujeres & \\
\hline$<^{1}$ & 12 & 5 & 17 \\
$1-14$ & 3 & 3 & 6 \\
$15-44$ & 10 & 5 & 13 \\
$45 y+$ & 18 & 8 & 26 \\
\hline Total & 43 & 19 & 62 \\
\hline
\end{tabular}

correspondiendo 12 a hombres, que es significativo en los resultados de la población mayor.

\subsection{Datos de la embarazada}

4.5.1 Distribución por grupo de edad.

Observamos que el mayor númera de muieres está entre los 20 a 24 años de edad, $33.2 \%$, seguido por las de 25 a 29 años, $24.8 \%$, encontrándose además que un $13 \%$ corresponde a las gestantes de 15 a 19 años, que es el grupo de edad calificado como de gran riesgo obstétrico. (Tabla No. 4).

Tabla No. 4

\section{EDAD (AÑOS) GESTANTES}

\begin{tabular}{|c|c|c|}
\hline Edad (Años) & No. & $\%$ \\
\hline $15-19$ & 56 & 13.0 \\
$20-24$ & 143 & 33.2 \\
$25-29$ & 107 & 24.8 \\
$30-34$ & 66 & 15.3 \\
$35-39$ & 38 & 8.8 \\
$40-44$ & 16 & 3.7 \\
$45-49$ & 3 & 0.7 \\
$50 y+$ & 2 & 0.5 \\
\hline Total & 431 & 100.0 \\
\hline
\end{tabular}

Tasa embarazo (15 a 44 años)

$=152.3 \times 10^{3}$ Mujeres

Tasa general de fecundidad $=107.2 \times 10^{3}$ Mujeres 


\subsubsection{Escolaridad de las gestantes}

El mayor número de pacientes sólo han hecho algún año de primaria $56.8 \%$. (Tabla No. 5).

Tabla No. 5

\begin{tabular}{|l|r|r|}
\hline $\begin{array}{l}\text { Escolaridad de } \\
\text { la Gestante }\end{array}$ & No. & \multicolumn{1}{c|}{$\%$} \\
\hline Analfabeta & 38 & 6.9 \\
Primaria & 314 & 56.8 \\
Secundaria & 175 & 31.6 \\
Universitaria & 12 & 2.2 \\
Carrera intermedia & 6 & 1.1 \\
Otras & 8 & 1.4 \\
\hline Total & 553 & 100.0 \\
\hline
\end{tabular}

4.5.3 Forma de terminación del Embarazo.

Tabla No. 6

\begin{tabular}{|l|r|r|}
\hline $\begin{array}{l}\text { Forma de terminación } \\
\text { del embarazo }\end{array}$ & No. & \multicolumn{1}{c|}{$\%$} \\
\hline Normal & 375 & 87.0 \\
Cesárea & 29 & 6.7 \\
Parto prematuro & 6 & 1.4 \\
Aborto 1er.trimestre & 16 & 3.7 \\
Aborto 2o. trimestre & 5 & 1.2 \\
Cesárea + parto prem. & & \\
\hline Total & 431 & 100.0 \\
\hline
\end{tabular}

* 146 probable al 81 embarazos
Se observó que el $87 \%$ de los partos fueron por vía vaginal, $6.7 \%$ partos in tervenidos por cesárea, además una baja incidencia de partos prematuros, $1.4 \%$.

La relación parto-aborto es de 20 a 1 , que se considera bastante baja con relación a la atención intra-hospitalaria en el Federico Lleras Acosta de Ibagué que es de 3 a 1 (Tabla No. 6).

\subsection{Control prenatal}

4.6.1 Lugar del control

Tabla No. 7

\begin{tabular}{|l|r|r|}
\hline Lugar control prenat. & No. & \multicolumn{1}{c|}{$\%$} \\
\hline Centro o Puesto de Salud & 153 & 35.4 \\
Casa & 7 & 1.5 \\
Hospital & 74 & 17.4 \\
I.S.S. & 74 & 17.4 \\
Médico Particular & 37 & 8.4 \\
Otros & 30 & 6.9 \\
No asisten & 56 & 13.0 \\
\hline Total & 431 & 100.0 \\
\hline
\end{tabular}

Se observa que el 35.4 de la población asiste a Puestos de Salud y el 17.4 al Hospital. Es de hacer notar que nuestro sistema de salud, por motivo de la sectorización de la Atención Materno Infantil, el Control Prenatal se hace en los Puestos de Salud, dejando al Hospital la atención de alto riesgo. (Tabla No. 7).

\subsubsection{Por trimestre.}

4.6.2.1 Gestantes sin control prenatal según trimestre de embarazo - 1980. 
Tabla No. 8

\begin{tabular}{|c|c|c|}
\hline Trimestre: & $\begin{array}{l}\text { No. gestantes que no } \\
\text { fueron a control }\end{array}$ & $\%$ \\
\hline I & 219 & 50.8 \\
II & 146 & 33.9 \\
III & 159 & 36.9 \\
\hline $\begin{array}{l}\text { Nunca fueron a } \\
\text { control }\end{array}$ & 33 & 7.6 \\
\hline
\end{tabular}

$\%$ Con base en 431 embarazadas

4.6.2.2 Promedio de controles prenatales -1980 .

Tabla No. 9

\begin{tabular}{|c|c|c|}
\hline Trimestre: & $\begin{array}{c}\text { No. gestantes } \\
\text { que fueron } \\
\text { a control }\end{array}$ & $\bar{x}$ Controles \\
\hline I & 212 & 1.9 \\
II & 285 & 2.2 \\
III & 272 & 2.3 \\
\hline
\end{tabular}

De acuerdo a los resultados, las pacientes empiezan a asistir en forma más regular en el segundo y tercer trimestre del embarazo, notándose en definitiva que las pacientes que deciden continuar control prenatal lo hacen mensualmente. (Tabla No. 8 y 9).

\subsubsection{Persona encargada.}

Es al médico a quién le corresponde el mayor número de controles prenatales, esto se debe a que en nuestro sistema de Salud en la población de Ibagué no se delegan funciones en el personal para- médico, además $14.2 \%$ de las pacientes embarazadas nunca acuden a ningún tipo de persona para su control, lo cual está mostrando que deben existir influencias que impiden aceptar los servicios de control prenatal, tales como situación económica, ocupación, tiempo, etc. (Tabla No. 10).

Tabla No. 10

\begin{tabular}{|l|r|r|}
\hline \multicolumn{1}{|c|}{$\begin{array}{c}\text { Persona encargada } \\
\text { Control prenatal }\end{array}$} & \multicolumn{1}{c|}{ No. } & \multicolumn{1}{c|}{ \% } \\
\hline Médico & 307 & 71.2 \\
Enfermera & 12 & 2.8 \\
Auxiliar & 6 & 1.4 \\
Partera & 7 & 1.6 \\
Médico y enfermera & 26 & 6.0 \\
Enfermera y auxiliar & 1 & 0.2 \\
Médico. Enferm. Aux. & 5 & 1.2 \\
Médico y Auxiliar & 6.4 & 1.4 \\
Nadie & 61 & 14.2 \\
\hline Total & 431 & 100.0 \\
\hline
\end{tabular}

\subsection{Complicaciones durante el Embarazo}

Tabla No. 11

\begin{tabular}{|l|r|r|}
\hline $\begin{array}{c}\text { Complicaciones durante el } \\
\text { embarazo }\end{array}$ & No. & $\%$ \\
\hline Hemorragia vaginal & 38 & 31.2 \\
Infecciones severas & 19 & 15.6 \\
Presión al ta y edema & 52 & 42.6 \\
H.Vaginal e Infecc. severas & 3 & 2.4 \\
H.Vaginal y presión alta & 6 & 4.9 \\
Infecc. severas, presión alta & 4 & 3.3 \\
\hline Total & 122 & 100.0 \\
\hline
\end{tabular}

Porcentaje complicaciones $=21.1$ 
La principal sintomatología asociada al embarazo corresponde a presión alta y edema, $42.6 \%$, seguida de la hemorragia genital, $31.2 \%$ y luego de las infecciones severas, $15.6 \%$. Hay que tener en cuenta que ese porcentaje seguirá siendo alto en la medida en que asistan o no al control prenatal. (Tabla No. 11).

\subsection{Datos sobre la atención parto y aborto}

4.8.1 Lugar de la atención

Tabla No. 12

\begin{tabular}{|l|r|r|}
\hline $\begin{array}{c}\text { Lugar de Atención del parto } \\
\text { o aborto }\end{array}$ & No. & \multicolumn{1}{c|}{$\%$} \\
\hline Centro o Puesto de Salud & 16 & 3.7 \\
Casa & 176 & 40.9 \\
Hospital & 149 & 34.6 \\
I.S.S. & 42 & 9.7 \\
Sala maternidad & 3 & 0.7 \\
Clínica particular & 37 & 8.6 \\
Otros & 8 & 1.8 \\
\hline Total & 431 & 100.0 \\
\hline
\end{tabular}

Se observa que el mayor número de partos son atendidos en casa, $40.9 \%$, superando al parto hospitalario, $34.6 \%$, lo que nos demuestra que hay varios factores que determinan que las pacientes no recurran a los servicios hospitalarios (educación - información - temor - error asistencial en el momento de la admisión de la paciente). (Tabla No. 12).

\subsubsection{Persona que atendió}

Relacionando los datos con el cuadro anterior, la atención del parto por parte- ra, auxiliar y enfermera es muy similar con el dato encontrado de parto atendido en casa, ya que en nuestro medio no existen los servicios de policlínica para atención del parto por personal paramédico. (Tabla No. 13).

Tabla No. 13

\begin{tabular}{|l|r|r|}
\hline Persona que la atendio & No. & \multicolumn{1}{c|}{$\%$} \\
\hline Médico & 209 & 48.5 \\
Enfermera & 44 & 10.2 \\
Auxiliar & 27 & 6.3 \\
Partera & 131 & 30.4 \\
Médico - Enfermera & 7 & 1.5 \\
Médico - Auxiliar & 2 & 0.5 \\
Otros & 11 & 2.6 \\
\hline Total & 431 & 100.0 \\
\hline
\end{tabular}

\subsubsection{Datos sobre el parto \\ 4.8.3.1 Tipo de parto}

Tabla No. 14

\begin{tabular}{|l|r|r|}
\hline Tipo de parto & No. & $\%$ \\
\hline Unico & 402 & 99.1 \\
Múltiple & 4 & 0.9 \\
\hline Total & 406 & 100.0 \\
\hline
\end{tabular}

La Tabla No. 14 muestra que la relación del parto múltiple (gemelar) único es de 1 a 99 y está de acuerdo con las estadísticas de Estados Unidos, que siguen la proporción a la fórmula establecida por HELIN. 


\subsection{Complicación materna post-parto, aborto}

4.9.1 Tipo de complicación

Tabla No. 15

\begin{tabular}{|c|c|c|}
\hline $\begin{array}{c}\text { Tipo de complicación } \\
\text { materna }\end{array}$ & No. & $\%$ \\
\hline Ninguna & 325 & 80.0 \\
\hline $\begin{array}{l}\text { Hemorragias durante parto o } \\
\text { aborto }\end{array}$ & 27 & 6.7 \\
\hline $\begin{array}{l}\text { Hemorragias post-parto o } \\
\text { aborto }\end{array}$ & 18 & 4.4 \\
\hline Desgarros & 18 & 4.4 \\
\hline Fiebre hasta 6 dias & 12 & 3.0 \\
\hline Muerte & 1 & 0.3 \\
\hline $5-3$ & & \\
\hline $2-3$ & 5 & 1.2 \\
\hline Total & 406 & 100.0 \\
\hline
\end{tabular}

La complicación materna más frecuente después del parto o aborto es la hemorragia.

4.9.2 Consultó después del parto o aborto.

Tabla No. 16

\begin{tabular}{|c|c|r|}
\hline $\begin{array}{l}\text { Consultó después del } \\
\text { aborto o parto }\end{array}$ & No. & \% \\
\hline SI & 137 & 31.8 \\
NO & 294 & 68.2 \\
\hline Total & 341 & 100.0 \\
\hline
\end{tabular}

Esta tabla nos muestra que el $68.2 \%$ de las pacientes que presentan parto 0 aborto no asisten a control médico posterior, que hace resaltar la falta de información sobre la importancia de los servicios posnatales.

\subsubsection{Lugar de la consulta}

Se observa que cuando acuden a control posterior, recurren a instituciones con servicios médicos, en primer lugar al Centro o Puesto de Salud $27.5 \%$ y en segundo lugar al Hospital $20.4 \%$ (Tabla No. 17).

Tabla No. 17

\begin{tabular}{|l|r|r|}
\hline $\begin{array}{l}\text { Lugar de consulta pos-parto } \\
\text { o aborto }\end{array}$ & No. & O \\
\hline Centro O.P. de Salud & 39 & 27.5 \\
Casa & 6 & 4.2 \\
Hospital & 29 & 20.4 \\
I.S.S. & 21 & 14.8 \\
Sala maternidad & 26 & 18.3 \\
Clínica particular & 21 & 14.8 \\
Otros & & \\
\hline Total & 142 & 100.0 \\
\hline
\end{tabular}

\subsection{Datos sobre el niño}

4.10.1 Edad de gestacion

Tabla No. 18

\begin{tabular}{|l|r|r|}
\hline Edad gestación niño & No. & \multicolumn{1}{c|}{$\%$} \\
\hline A término & 391 & 95.0 \\
$7-8$ meses & 20 & 5.0 \\
\hline Total & 411 & 100.0 \\
\hline
\end{tabular}


La tabla que relaciona la edad gestacional del niño nos muestra que en nuestro medio la incidencia de la prematurez es del $5 \%$, similar a estadísticas de otros países con características socioeconómicas iguales a las nuestras ( $\mathrm{Ta}$ bla No. 18).

\subsubsection{Presentación}

Tabla No. 19

\begin{tabular}{|l|r|r|}
\hline Presentacion niño & No. & $\%$ \\
\hline De cabeza & 389 & 95.8 \\
\hline De pelvis & 14 & 3.5 \\
\hline Otra & 2 & 0.5 \\
\hline Sin datos & 1 & 0.2 \\
\hline Total & 406 & 100.0 \\
\hline
\end{tabular}

La proporción de partos en presentación de pelvis es de $3.5 \%$ en relación a todos los partos y está de acuerdo a estudios realizados en los Estados Unidos en la Universidad de .GEORGETOWN. (Tabla No. 19).

\subsubsection{Complicación presentada}

Esta tabla nos muestra que la principal complicación del recién nacido es el síndrome de insuficiencia respiratoria, por diferentes causas tales como prematurez, bronco-aspiración, etc. (Tabla No. 20).

\subsection{El niño vive actualmente}

\subsubsection{Control del niño sano}

Esta tabla nos indica que el $63 \%$ de los recién nacidos no son llevados a control médico. (Tabla No. 21).
Tabla No. 20

\begin{tabular}{|l|r|r|}
\hline Complicación del niño & No. & \multicolumn{1}{c|}{ \% } \\
\hline Demora en respirar & 20 & 4.9 \\
Cianótico & 28 & 6.9 \\
Fue hospitalizado & 4 & 1.0 \\
Dem. resp, cianótico, hospi & & \\
tal & 8 & 2.0 \\
Demora respirar, cianótico & 20 & 4.9 \\
Demora respirar, hospi- & 2 & 0.5 \\
talizado & 2 & 0.5 \\
Cianótico hospitalizado & 316 & 77.8 \\
Ninguna & 6 & 1.5 \\
Sin dato & 406 & 100.0 \\
\hline Total
\end{tabular}

Tabla No. 21

\begin{tabular}{|c|c|r|}
\hline Control niño sano & No. & $\%$ \\
\hline SI & 151 & 37.2 \\
NO & 255 & 62.8 \\
\hline Total & 406 & 100.0 \\
\hline
\end{tabular}

\section{RESUMEN Y CONCLUSIONES}

Se hace revisión Estadística de 2.867 familias con un promedio de 5.6 personas por familia.

Se obtuvieron las siguientes tasas de mortalidad: Mortalidad General $4 \times$ 1.000. Mortalidad infantil $41.9 \times 1.000$, muy parecida a la encontrada para el Departamento del Tolima en 1980 $(40 \times 1.000)$. El porcentaje de muertes en menores de un año con respecto al total de muertes fue de 27.4. La mortalidad materna hallada fue de $2.5 \times 1.000$. 
Se encontraron las siguientes:

Embarazo $117.2 \times 1.000$, fecundidad general $107.2 \times 1.000$, natalidad 20.2 $\times 1.000$, morbimortalidad $7.3 \times 1.000$.

El porcentaje de mujeres en eaad reproductiva (15 a 44 años) con respecto a la población total fue de $24.6 \%$.

Las complicaciones más frecuentes del embarazo son presión alta y edema $(42.6 \%)$ y hemorragia vaginal $(31.2 \%)$. El porcentaje de complicaciones durante el embarazo fue de $21.1 \%$. La condición patológica puerperal más frecuente fue la hemorragia genital.

El promedio de partos por mujer es de 2.9. Y la tasa específica de embarazo es re $152.3 \times 1.000$.

Fue evidente que a pesar de que el mayor número de las embarazadas asistieron a uno cualquiera de los Centros para Control Prenatal, tuvieron el parto en casa, lo que revela la falta de información, remisión al Centro Hospitalario.

El número de nacidos vivos fue de 406 que da una tasa de natalidad de 26.3 $x$ 1.000. El crecimiento vegetativo de la población hallado fue de $22.3 \times 1.000$.

\section{RECOMENDACIONES}

6.1 Lograr pleno aprovechamiento de los recursos disponibles, tanto humanos como institucionales; reconsiderar las asignaciones de recursos para conseguir, en forma equitativa, el gradual aumento de la cobertura de la población, excediendo al incremento progresivo de la misma.

6.2 El análisis de la encuesta presentada ofrece para el conocimiento una realidad que nos permite prever posibles situaciones futuras, condicionándolas por medio de decisiones de política que desde ahora ordenen $y$ faciliten los cambios $y$ transformaciones necesarias para el logro del bienestar materno-fetal.

6.3 Conseguir la participación activa de las Sociedades de Obstetricia y Pediatría en los programas relacionados con la salud materno infantil.

6.4 Activar y mejorar los programas de educación a la madre tanto en grupo como individualmente por intermedio del personal paramédico.

\section{RESEARCH ON PERINATAL MORBIDITY AND MORTALITY RATES IN SUBURBAN SLUMS OF THE CITY OF IBAGUE}

\section{Summary and conclusions}

2.867 families were studied and an average of 5.6 people per family was found.

The following mortality rates were established: General mortality: 4 x 1.000. Infant mortality: $41.9 \times 1.000$, quite similar to the rate for the whole Department of Tolima in 1980 (40 x 1.000). The percentage of deaths in children below one year in relation to total deaths was 27.4. Maternal mortality was found to be $2.5 \times 1.000$.

Other rates found were: Pregnancy: $117.2 \times 1.000$. General fertility: $107.2 \times$ 1.000. Birth: $20.2 \times 1.000$. Morbiditymortality: $7.3 \times 1.000$. The percentage of women in reproductive age (15-44) in relation to the total population was 24.6 . 
High blood pressure and edema are the most frequent complications during pregnancy $(42.6 \%)$, followed by vaginal bleeding $(31.2 \%)$. The total percentage of complications during pregnancy was found to be $21.1 \%$. The most frequen puerneral pathology was genital bleeding.

For every women the average births are 2.9 and the specific pregnancy rate was $152.3 \times 1.000$.

\section{Bibliografía}

1. R.R. PUFFER Y C.V. SERRANO, “Results of the inter-American investigations of mortality relating to reproduction". En Epidemology of abortion and practcs of fertility regulation in latin America; Seleted reports (PA.HO Publicación Científica No. 306, 1975).

2. SUSAN SCRIMSHAWETAL, "Aceptabilidad e impacto: $L a$ evaluación en un programa modelo de prestación de servicios de salud en Cali, Colombia, Nueva York: Universidad de Columbia, Instituto Internacional para el estudio de la reproducción humana, 1975.

3. SUSAN CH. SCRIMAHAW, "La mortalidad Infantil y el comportamiento respecto a la regulación del tamaño de la
It became evident that in spite of the fact that most pregnant women attended the antenatal care centers, they had their deliveries at home, which reflects either lack of information or lack of adequate referral to a hospital.

The number of live births was 406, which gives a birth rate of $26.3 \times 1.000$ Natural population growth was $22.3 \times$ 1.000 .

4. JUAN PABLO TERRA, consultor de Unicef. "Situación de la Infancia en América Latina y el Caribe". Estudios de población número especial abril de 1980 pág. $45-80$.

5. BEHM HUGO, material de enseñanza sobre mortalidad con especial referencia a la situación de América Látina, Celade.

6. Evaluación del programa materno infantil de Colombia 1974 - 76. Julio de 1976. Ministerio de Salud OPS, fondo de las Naciones Unidas para actividades de población.

7. Ministerio de Salud. Boletín epidemiológico nacional. Julio - septiembre 1977 Vol. 3 No. 3 - 4 . 